\title{
Absorção de água e propriedades mecânicas de compósitos poliméricos utilizando resíduos de MDF
}

\section{Water absorption and mechanical properties of polymer composites using waste MDF}

\author{
Jailton Weber Gomes ${ }^{1 *}$, Glauber Silva Godoi², Luiz Guilherme Meira de Souza ${ }^{3}$ e \\ Luiz Guilherme Vieira Meira de Souza ${ }^{3}$ \\ 'Instituto Federal da Bahia - IFBA, Camaçari, BA, Brasil \\ 2Laboratório de Química Interfacial e de Materiais - LABQUIM, Departamento de Química, \\ Universidade Federal de Sergipe - UFS, São Cristóvão, SE, Brasil \\ ${ }^{3}$ Departamento de Engenharia Mecânica, Universidade Federal do Rio Grande do Norte - UFRN, \\ Natal, RN, Brasil \\ *jailtonweber@ifba.edu.br
}

\begin{abstract}
Resumo
Apresenta-se o estudo de viabilidade de utilização, como reforço em materiais compósitos poliméricos, de resíduos originados da manufatura dos painéis de MDF (Medium Density Fiberboard), avaliando seu potencial como alternativa em substituição a cargas minerais para aplicação industrial. A escolha da resina poliéster ortoftálica foi motivada pelas suas propriedades e principalmente pelo seu baixo custo e facilidade de aquisição. Diferentes frações mássicas $(m)$ de adição de resíduo em resina poliéster ortoftálica foram estudadas: 0, 10, 15 e 20\%. As propriedades analisadas foram: resistência mecânica, absorção de água e grau de cristalinidade. Os resultados mostram a diminuição das propriedades mecânicas em até $53 \%$, no índice de cristalinidade em até $18 \%$ e aumento no índice de absorção de água em quase cinco vezes para o compósito de maior adição de resíduo (C20).
\end{abstract}

Palavras-chave: compósitos poliméricos, resíduos de $M D F$, resina poliéster ortoftálica, resistência mecânica, índice de cristalinidade.

\begin{abstract}
The study examines the feasibility of using MDF (Medium Density Fiberboard) waste as reinforcement in orthophthalic polyester resin, evaluating its potential as an alternative to mineral filler for industrial applications. The orthophthalic resin was chosen because it's good performance, low cost and easily obtainable. Three composite compositions were prepared containing different amount of waste MDF (10,15 and 20\%). The mechanical properties, degree of crystallinity and water absorption of the resin and its respective composites were evaluated. The results show a decrease in mechanical strength and crystallinity index followed by an increase in water absorption index caused by poor adhesion in the region of the polymer matrix fiber interface.
\end{abstract}

Keywords: polymeric composites, MDF waste, polyester resin orthophthalic, mechanical strength, crystallinity index.

\section{Introdução}

O MDF, do inglês Medium Density Fiberboard, ou Chapa de Fibra de Média Densidade, é um importante compósito preparado a partir de fibras lignocelulósicas, oriundas normalmente de madeira, que são misturadas com resinas sintéticas e posteriormente submetidas à prensagem em altas temperaturas o que confere ao produto o típico formato de chapas com densidade na faixa de $500-900 \mathrm{~kg} / \mathrm{m}^{3[1]}$. As resinas sintéticas mais usadas são à base de uréia-formaldeído, tanino-formaldeído e melanina-uréia-formaldeído, sendo esta última bastante usada quando se deseja maior resistência à umidade $^{[2,3]}$. Além destes materiais, outros podem ser usados a depender da propriedade de interesse tais como retardantes de chama, fungicidas, entre outros ${ }^{[1,2]}$.

O MDF é um material uniforme, livre de nós, e de superfície lisa e plana. Estas características o tornam de fácil usinagem, além de apresentar melhor rendimento da matéria prima e preço mais acessível quando comparado à madeira natural. Estas características o tornaram popular em diversos segmentos, tais como na fabricação de móveis, na construção civil e de peças de artesanato em substituição ao uso da madeira natura ${ }^{[1,3]}$. Mesmo em tempos de crise, estudos de mercado indicam o crescimento da indústria 
de painéis, principalmente o MDF, nos próximos anos no Brasil e em outros países ${ }^{[4,5]}$.

Um aspecto importante a ser destacado sobre o processo produtivo de corte do MDF nas marcenarias e na indústria moveleira é a grande quantidade de resíduo na forma de um pó fino constituído principalmente de fibras lignocelulósicas (Figura 1). Diante deste desafio, é importante que as empresas e indústrias do setor realizem a implantação de sistemas certificáveis de gestão ambiental, como a norma ISO 14001, viabilizando uma adequada gestão de resíduos que permita obter benefícios sociais, ambientais econômicos ${ }^{[-8]}$. Como exemplo podemos citar a utilização do resíduo em aplicações diversas tais como: fabricação de painéis aglomerados, tijolos e compósitos poliméricos; combustível alternativo para geração termoelétrica; produção de produtos de maior valor agregado através de conversão térmica; entre outras ${ }^{[9-20]}$.

Infelizmente existe ainda no Brasil casos em que o descarte ocorre de maneira aleatória na natureza, resultando em grave impacto ambiental devido principalmente a característica particulada deste resíduo, favorecendo a liberação para a atmosfera de compostos orgânicos voláteis (em inglês, Volatile Organic Compounds, VOCs), especialmente o formaldeído, uma substância comprovadamente cancerígena de acordo com a Agência Internacional de Pesquisa em Câncer (International Agency for Cancer Research, IARC) ${ }^{[21]}$. A liberação de formaldeído na atmosfera pode ser causada por uma eventual hidrólise da resina uréia-formaldeído presente no resíduo quando exposto a umidade ${ }^{[22]}$ ou por causa da queima indevida do resíduo para fins energéticos (geração termoelétrica). Por este motivo é importante ressaltar que o empreendimento que deseje utilizar este resíduo como combustível cumpra os requisitos da portaria estadual pertinente além de possuir licença ambiental específica ${ }^{[23]}$.

Os compósitos poliméricos têm se apresentado como uma alternativa importante para o aproveitamento de resíduos de madeira e de MDF em matrizes de resinas termoplásticas, também conhecidos como compósitos plásticos de madeira (Wood Plastic Composite, WPC), ou de resinas termofixas ${ }^{[14,18,24,25]}$.

Compósitos confeccionados com polietileno de alta densidade "virgem" (v-HDPE) e diferentes tipos de serragem gerados na indústria moveleira foram produzidos por

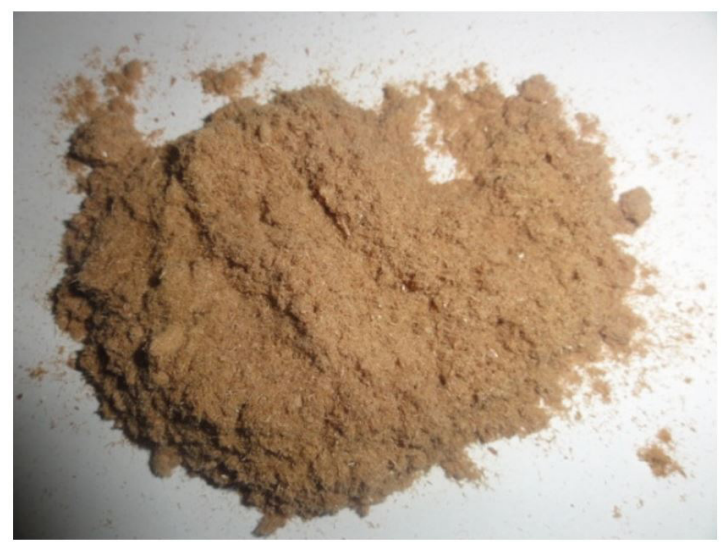

Figura 1. Resíduo de MDF. extrusão onde foram utilizados resíduos de madeira pinus (Pinus taeda), de aglomerado, de MDF e de madeira de eucalipto (Eucalyptus grandis), juntamente com um agente de acoplamento à base de anidrido maléico. Os compósitos fabricados com MDF e aglomerados apresentaram as menores resistências mecânicas ${ }^{[14]}$.

Em outro exemplo de compósito também preparado com resina termoplástica, cloreto de polivinila (Polyvinyl chloride, $P V C$ ) foi misturado com resíduo de madeira pinus em diversas quantidades. Os resultados mostram que o desenvolvimento deste tipo de material compósito pode substituir o uso da madeira natural em diversas aplicações como em deck de piscinas e fabricação de bancos de praças $^{[26]}$.

Compósitos produzidos em matriz de resina termofixa de poliéster insaturado foram preparados pela mistura com resíduos de madeira oriundos de máquinas de serra do tipo fita e industrial, furadeira, plaina e desempenadeira. Ensaios de absorção de água, ensaio de dureza Shore D e ensaio de flexão em três pontos foram realizados para demonstrar sua viabilidade de utilização. Houve aumento na absorção de água conforme se adiciona os resíduos, porém em níveis muito melhores que a da madeira sólida. Quanto à dureza, o resíduo diminuiu cerca de $4 \%$ tal propriedade em relação à matriz, resultado considerado pouco significativo, porém a rigidez aumentou em torno de $27 \%$ para a adição de $10 \%$ e $22 \%$ para adição de $20 \%$ de resíduo ao compósito. Houve também modificação do aspecto físico estético como a cor e textura ${ }^{[19]}$.

Neste trabalho, resíduos de MDF em pó foram empregados na preparação de compósitos em matriz de resina termofixa de poliéster ortoftálica. Três composições contendo diferentes frações mássicas $(\mathrm{m})$ de resíduo em resina poliéster ortoftálica foram estudadas: 10, 15 e 20\% $\mathrm{m} / \mathrm{m}$. As propriedades analisadas da resina e seus respectivos compósitos foram: resistência mecânica, grau de cristalinidade e absorção de água.

\section{Materiais e Métodos}

\subsection{Materiais}

O resíduo utilizado (Figura 1) apresenta-se na forma de serragem oriunda das operações de corte com serra circular de painéis de MDF usados na fabricação de móveis. Como o objetivo é o aproveitamento deste resíduo na forma coletada não foi feita análise de granulométrica.

A matriz utilizada para fabricação dos compósitos foi a resina poliéster ortoftálica de baixa reatividade e pré-acelerada (Ashland Arazyn 1.0). Esta escolhida foi motivada pelos seguintes fatores: facilidade de aquisição em qualquer loja especializada em manutenção de piscinas, vasta aplicação em diversos segmentos industriais e seu baixo custo. O peróxido de metiletilcetona (Methyl Ethyl Ketone Peroxide, MEKP) foi utilizado como elemento ativador de polimerização na quantidade de $1,0 \%$ da massa de resina utilizada. As propriedades físicas da resina podem ser vistas na Tabela 1.

Para facilitar a retirada das placas de compósitos fabricadas pelo método de moldagem por compressão foi utilizado um desmoldante a base de cera de carnaúba, um 
produto facilmente adquirido em lojas especializadas em manutenção de piscinas ou de materiais de construção.

A água utilizada para ensaio de absorção foi adquirida em posto de gasolina. Mas existe a possibilidade de aquisição em lojas de autopeças.

Prensa hidráulica com capacidade de 5 toneladas foi utilizada para prensagem do compósito acondicionado em molde de ferro fundido com dimensão de $200 \mathrm{~mm}$ x $200 \mathrm{~mm}$ x $15 \mathrm{~mm}$ (comprimento $\mathrm{x}$ largura $\mathrm{x}$ altura).

Garrafas de politereftalato de etileno (PET) devidamente reaproveitadas foram utilizadas como recipientes para misturas durante a fabricação dos compósitos.

\subsection{Procedimentos para fabricação do compósito}

As amostras de compósitos com diferentes teores de resíduo (\% em massa) foram as seguintes: $\mathrm{C}$ (sem acréscimo de resíduo), C10 (10\%), C15 (15\%) e C20 (20\%). O teor máximo de adição de resíduo foi de $20 \%$, pois a partir desta concentração há dificuldade de homogeneização pelo processo manual.

O procedimento de obtenção do compósito de forma manual está representado no fluxograma da Figura 2.

Primeiramente deve-se aplicar desmoldante no molde o qual dará origem as placas de onde serão extraídos os corpos de prova para os ensaios. O tempo de secagem após aplicação do desmoldante é de 30 minutos. Importante aplicar pelo menos três camadas.

O único tratamento imposto ao resíduo foi o peneiramento. Por se tratar de um resíduo este processo é importante para diminuir o risco de adição de impurezas na confecção dos compósitos. Para este procedimento utilizou-se uma peneira granulométrica em aço inox, MESH/TYLER 9 com abertura de $2 \mathrm{~mm}$.

Antes da mistura dos componentes do compósito, foi realizada a adição do acelerador de polimerização da resina, o peróxido de metiletilcetona (Metyl Ethyl Ketone Peroxide, MEKP) na quantidade de $1,0 \%$ em relação a massa total do compósito a ser preparado. Esta adição deve ser feita em pequenas quantidades, garantido a melhor homogeneidade da mistura. Após aproximadamente 2 minutos de homogeneização foi adicionada a quantidade apropriada de resíduo de MDF.

Tabela 1. Principais propriedades físicas da resina poliéster ortoftálica empregada como matriz do compósito polimérico proposto.

\begin{tabular}{lc}
\hline \multicolumn{1}{c}{ PROPRIEDADES DA RESINA AZ 1.0 - PADRÃO } \\
\hline Tempo de gel & $10-14 \mathrm{~min}$ \\
Pico Exotérmico & Max. $150^{\circ} \mathrm{C}$ \\
Aspecto & Cristal \\
Cor depois de curada & Incolor \\
Resistência a Tração & $57,0 \mathrm{MPa}$ \\
Módulo de Tração $^{[27]}$ & $1970 \mathrm{MPa}$ \\
Alongamento & $3,2 \%$ \\
Resistência a Flexão $^{[28]}$ & $84,4 \mathrm{MPa}$ \\
Módulo na Flexão $^{[28]}$ & $2814,3 \mathrm{MPa}$ \\
\hline Normas utilizadas: ASTM D-638
\end{tabular}

O processo de prensagem deve acontecer após 15 minutos da adição do MEKP, pois esse é o tempo de gel médio para o início de polimerização. É importante aguardar o tempo de gel, caso contrário a resina pura pode sair pelas frestas do molde não ocorrendo a prensagem devidamente. A pressão de conformação usada foi de aproximadamente $370 \mathrm{kPa}, \mathrm{o}$ que conferiu um bom grau de compactação da placa sem risco de danificar o molde. A retirada do molde da prensa ocorreu três horas após a prensagem, todavia a retirada da placa fabricada deve ocorrer no mínimo 6 horas após início do processo de fabricação da placa. Após a retirada da placa foi realizado o procedimento de pós-cura por 2 horas à $80^{\circ} \mathrm{C}$ conforme recomendação do fabricante.

É importante destacar ainda que foi tomado um cuidado especial no que diz respeito ao acabamento superficial dos corpos de prova obtidos a partir das placas inicialmente prensadas. O objetivo do lixamento e do polimento realizados foi de eliminar eventuais defeitos superficiais que pudessem servir como concentradores de tensão, prejudicando as propriedades obtidas nos ensaios mecânicos.

\subsection{Corpos de prova}

A Tabela 2 apresenta as dimensões dos corpos de prova fabricados. Para os ensaios mecânicos as dimensões foram definidas conforme as normas da American Society for Testing and Materials ${ }^{[27-29]}$.

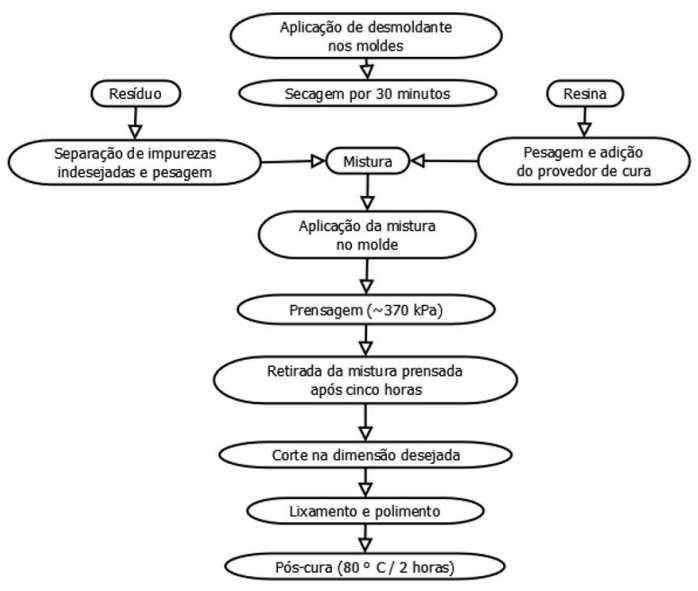

Figura 2. Fluxograma de etapas e procedimentos para fabricação do compósito.

Tabela 2. Dimensões dos corpos de prova.

\begin{tabular}{lcc}
\hline \multicolumn{1}{c}{ Tipo de Ensaio } & $\begin{array}{c}\text { Dimensões } / \mathbf{m m} \\
(\mathbf{C} \times \mathbf{L} \times \mathbf{E})\end{array}$ & $\begin{array}{c}\text { Amostras } \\
\text { analisadas }\end{array}$ \\
\hline Tração $^{[27]}$ & $250 \times 25 \times 8$ & 10 \\
Flexão $^{[28]}$ & $250 \times 25 \times 8$ & 10 \\
$D R X$ & $25 \times 25 \times 8$ & 1 \\
MEV & $15 \times 15 \times 8$ & 1 \\
Absorção de água ${ }^{[2]}$ & $50 \times 50 \times 3$ & 5 \\
\hline
\end{tabular}

Normas utilizadas: ASTM D-638 $8^{[27]}$, ASTM D-790[28], ASTM D-570 ${ }^{[29]} ; \mathrm{C}=$ Comprimento; $\mathrm{L}=$ Largura; $\mathrm{E}=$ Espessura. 


\subsection{Caracterização}

As seguintes técnicas de caracterização foram empregadas:

Medida de pH - Foi realizada apenas para o resíduo e feita indiretamente através da medida de $\mathrm{pH}$ de uma solução aquosa de resíduo ( $10 \%$ em massa) após 6 horas de preparo. A medida foi feita utilizando papel indicador de $\mathrm{pH}$ (Merck).

Microscopia Eletrônica de Varredura - As análises do resíduo, do MDF e dos compósitos preparados foram realizadas em um microscópio eletrônico de varredura modelo TESCAN VEGA3. Foram analisadas as superfícies de fratura dos corpos de prova previamente rompidos nos ensaios de tração e flexão. Os experimentos ocorreram a uma temperatura ambiente de $23{ }^{\circ} \mathrm{C}$ e umidade relativa de $65 \%$.

Difração de Raios-X- Os difratogramas foram obtidos à temperatura ambiente em difratômetro de Raios-X modelo Shimadzu modelo XRD-7000 usando radiação $\mathrm{Cu} \mathrm{K \alpha}$ $(\lambda=0,15418 \mathrm{~nm} ; \mathrm{I}=30 \mathrm{~mA} ; \mathrm{V}=40 \mathrm{kV})$ no intervalo de $2 \theta$ variando de $5^{\circ}$ a $90^{\circ}$ e passo de $2^{\circ} \mathrm{min}^{-1}$. A Equação 1 foi utilizada para o cálculo do grau de cristalinidade baseado no Método Relativo ${ }^{[30]}$.

$$
I_{c} \%=\frac{P_{(c r)}-P_{(a m)}}{P_{(c r)}} \times 100
$$

Onde:

$\mathrm{I}_{\mathrm{c}}=$ Índice de cristalinidade em porcentagem;

$\mathrm{I}_{\mathrm{cr}}=$ Pico de intensidade de difração que representa o material cristalino;

$\mathrm{I}_{\mathrm{am}}=$ Pico de intensidade de difração que representa o material amorfo;

Ensaios mecânicos de flexão e tração - Foi utilizada a máquina universal modelo WDW-20 E série 271 para a determinação das propriedades mecânicas (resistência à tração e flexão, módulo de elasticidade na tração e na flexão e alongamento total na tração e na flexão) dos corpos de prova.

Absorção de água - Foram utilizados 5 pastilhas de cada material, cujas dimensões estão na descritas na Tabela 2. Os procedimentos foram baseados na ASTM 570-98 ${ }^{[29]}$. Utilizou-se a Equação 2 para determinação do percentual de água absorvida, seja destilada ou água do mar.

$$
\text { Abs }=\frac{P_{f}-P_{i}}{P_{i}} \times 100
$$

Onde:

Abs: Absorção de água em \%;

$\mathrm{Pi}=$ Massa inicial;

$\mathrm{Pf}=$ Massa final

\section{Resultados e Discussões}

\section{1 Medida de $\mathrm{pH}$}

A medida de $\mathrm{pH}$ do resíduo apresentou resultado neutro. O objetivo desta medida foi de verificar se eventuais valores ácidos ou básicos poderiam causar um aceleramento ou retardamento da cura da resina, o que parece não ter ocorrido. Em um estudo sobre o efeito do $\mathrm{pH}$ e do catalisador na cura de resina uréia-formaldeído, Xing et al. ${ }^{[31]}$ observaram que a acidez da fibra pode reduzir o tempo de cura da resina principalmente em condições de baixos teores de catalisador na mistura.

\subsection{Microscopia eletrônica de varredura}

A Figura 3a mostra a superfície de fratura de uma chapa de MDF, preparada a partir do resíduo, onde pode ser verificada a estrutura fibrosa típica formada pelas fibras lignocelulósicas. Outro detalhe importante mostrado na Figura 3 b é o formato achatado das fibras de eucalipto.

$\mathrm{Na}$ análise do resíduo não foi observado nas regiões analisadas vestígios de outros materiais ou contaminantes que pudessem interferir no processamento do compósito. Uma característica importante observada foi a presença de aglomerados e de certa heterogeneidade nas características anatômicas das fibras que compõe o resíduo. A diferença no comprimento, por exemplo, pode atingir uma ordem de grandeza, com fibras variando de aproximadamente $300 \mu \mathrm{m}$ até $3 \mathrm{~mm}$ (Figura 3c). O processo de desfibramento dos cavacos de madeira ${ }^{[32]}$ usados na produção das chapas de MDF bem como o próprio processo produtivo de corte das chapas fabricadas podem produzir fibras descontínuas, e
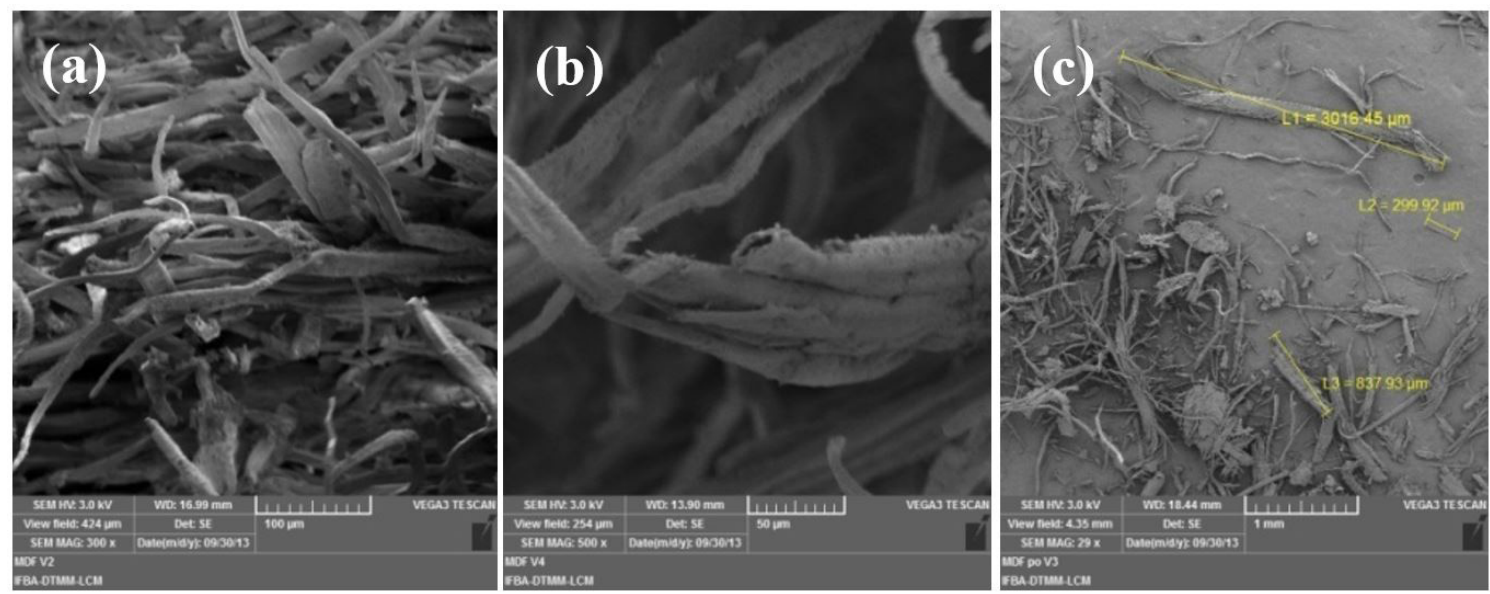

Figura 3. Micrografias obtidas por MEV: (a) superfície de fratura da amostra de resíduo de MDF prensado; (b) Detalhe da fibra na amostra prensada; (c) Resíduo de MDF na forma original (pó). 

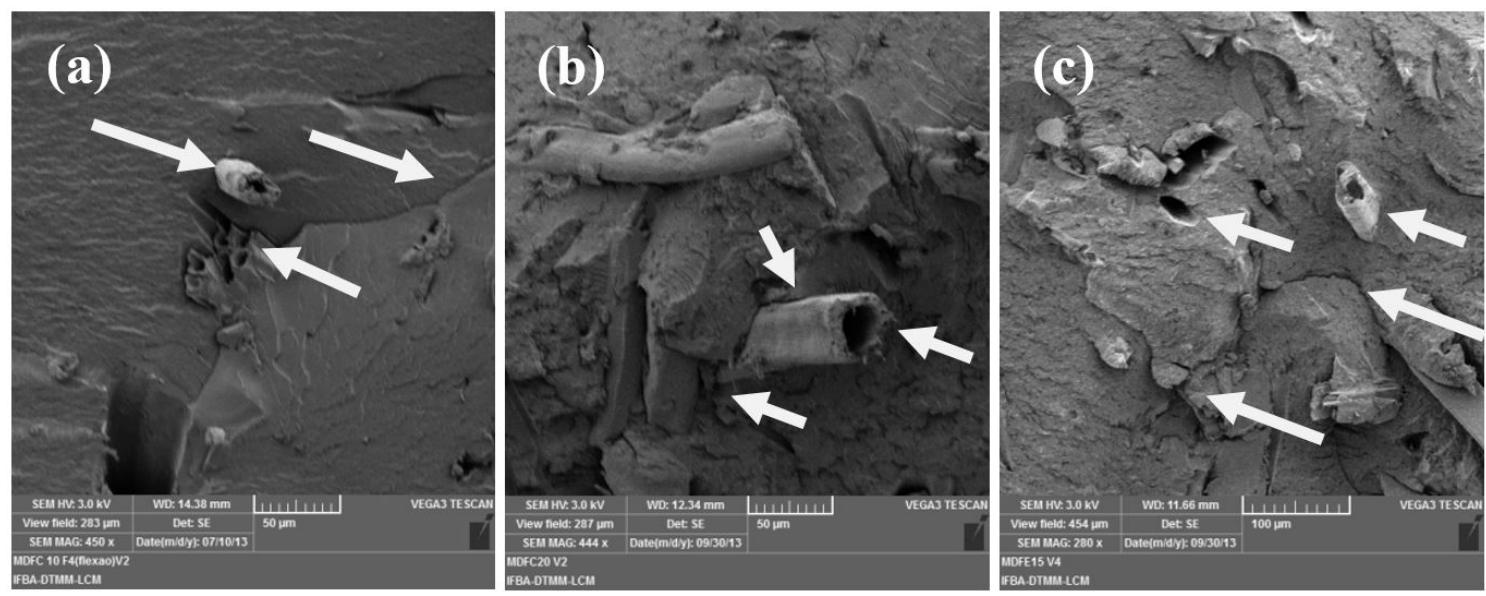

Figura 4. Micrografias obtidas por MEV dos compósitos preparados:(a) C10; (b) C15 e (c) C20.

portanto, com diferenças no comprimento. Outra hipótese que deve ser considerada é que a chapa de MDF tenha sido fabricada a partir da mistura de fibras de espécies coníferas, de comprimento longo, e de fibras de espécies folhosas, principalmente o eucalipto, de comprimento mais curto ${ }^{[1]}$. Esta é uma tendência natural no mercado de produção de MDF tendo em vista a maior competitividade das florestas de eucalipto ${ }^{[8]}$, apesar de alguns aspectos ambientais controversos relativos a esta monocultura ${ }^{[33-36]}$.

As Figura 4a, b, c apresentam as micrografias dos compósitos preparados. Na Figura 4a é possível observar fibras com características anatômicas bastante distintas, tais como o índice de Runkel e fração parede ${ }^{[37,38]}$, o que parece confirmar a hipótese de mistura de fibras de espécies coníferas e folhosas no resíduo de MDF.

A presença de pull out, um importante mecanismo de falha caracterizado pelo desprendimento das fibras da matriz durante a fratura, foi verificado em todos os compósitos conforme mostrado nas Figura 4a, b, c. Outra característica importante mostrada nas Figura 4b, c são as fissuras ou fraturas coesivas na matriz. Um aspecto importante observado na microestrutura de todos os compósitos é a distribuição aleatória das fibras na matriz. Na Figura 4b, por exemplo, é possível observar uma fibra orientada na direção da fratura, o que não contribui para o mecanismo de reforço inicialmente proposto no trabalho. Estas características são responsáveis pela deterioração das propriedades mecânicas observadas nos compósitos. Na Figura 4c, por exemplo, é possível observar a presença de poros possivelmente deixados na matriz devido ao desprendimento das fibras já mencionado.

Em todas as fibras analisadas nos compósitos não foi verificada a penetração da resina em sua cavidade interna, o que pode contribuir para uma menor adesão junto a matriz bem como para a absorção de água.

\subsection{Difração de Raios-X}

O difratograma de Raios-X mostrado na Figura 5a corresponde à amostra sólida de MDF preparada a partir da prensagem do resíduo. Os principais picos localizados em valores aproximados de $2 \theta \mathrm{em} 14,9^{\circ}, 16,7^{\circ}, 22,8^{\circ} \mathrm{e}$
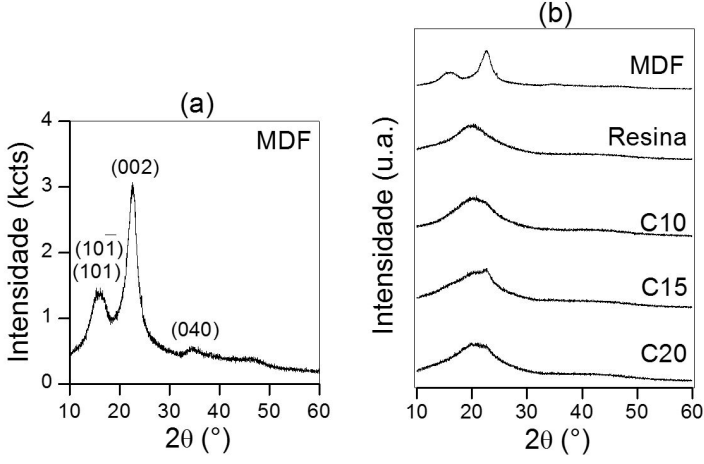

Figura 5. (a) Difratograma do resíduo MDF e (b) comparativo dos difratogramas do MDF com os da resina cristal e compósitos preparados.

$34,9^{\circ}$ são posições características de reflexões dos planos cristalográficos (101), (10 $\overline{1}),(002)$ e (004) da celulose tipo $\mathrm{I}^{[39]}$, que é uma forma polimórfica da celulose de ocorrência natural. Este é um resultado esperado já que as fibras lignocelulósicas são o principal constituinte estrutural do MDF.

No difratograma da resina mostrado na Figura $5 \mathrm{~b}$ pode ser observado a presença de dois picos importantes. $\mathrm{O}$ primeiro em aproximadamente $21^{\circ}$, que corresponde à fase cristalina defeituosa, e o segundo em $41^{\circ}$, correspondente a parcela amorfa da matriz pura sem adição de resíduo, provavelmente devido à má formação da cadeia polimérica na cristalização do polímero.

A partir da análise da intensidade relativa destes picos nos difratogramas das amostras mostradas na Figura $5 \mathrm{~b}$ foi possível a determinação do grau de cristalinidade ${ }^{[30]}$ da resina cristal e dos compósitos preparados conforme mostrado na Tabela 3. O valor obtido para a resina foi de $72,41 \%$, ligeiramente superior ao obtido para os compósitos que foi de aproximadamente $60 \%$ para todos os compósitos. Estes dados nos permitem concluir que o teor de resíduo não promoveu mudança significativa no grau de cristalinidade da matriz em todos compósitos. 
Tabela 3. Cristalinidade do Compósito.

\begin{tabular}{cccccc}
\hline Amostra & Ic (u.a.) & Iam (u.a.) & $\mathbf{2 \theta}$ & 20am & Ic (\%) \\
\hline$C$ & 2160 & 596 & 20,3 & 72,41 & 29,7 \\
$C 10$ & 1928 & 714 & 22,7 & 29,1 & 29,5 \\
$C 15$ & 1880 & 734 & 21,0 & 60,95 \\
$C 20$ & 1676 & 682 & 22,3 & 29,1 \\
\hline
\end{tabular}

Tabela 4. Resultados obtidos no ensaio de tração e flexão.

\begin{tabular}{|c|c|c|c|c|c|c|}
\hline \multirow{2}{*}{ Amostra } & \multicolumn{2}{|c|}{ Tensão de Resistência (MPa) } & \multicolumn{2}{|c|}{ Alongamento Total (\%) } & \multicolumn{2}{|c|}{ Módulo de Elasticidade (Gpa) } \\
\hline & Tração & Flexão & Tração & Flexão & Tração & Flexão \\
\hline$C$ & 39,06 & 3,83 & 2,91 & 22,64 & 1,26 & 0,02 \\
\hline$C 10$ & 19,19 & 2,68 & 1,36 & 2,25 & 2,07 & 0,12 \\
\hline$C 15$ & 18,63 & 2,60 & 1,23 & 1,89 & 2,10 & 0,15 \\
\hline$C 20$ & 18,39 & 2,51 & 1,10 & 1,53 & 2,20 & 0,19 \\
\hline
\end{tabular}

Tabela 5. Absorção de água destilada e do mar (\% em massa).

\begin{tabular}{cccc}
\hline Meio & \multicolumn{3}{c}{ Amostra } \\
\cline { 2 - 4 } & C & C10 & C15 \\
\hline Água destilada & $0,998 \pm 0,033$ & $2,059 \pm 0,052$ & $4,336 \pm 0,052$ \\
Água do mar & $1,107 \pm 0,696$ & $3,094 \pm 0,076$ & $6,631 \pm 0,092$ \\
\hline
\end{tabular}

\subsection{Ensaios mecânicos de tração e flexão}

A Tabela 4 apresenta os resultados obtidos no ensaio de tração e flexão para as amostras analisadas. A amostra sem adição de resíduo (resina poliéster) apresentou valores de resistência mecânica a tração e a flexão inferiores aos apresentados na Tabela 1, que são os valores fornecidos pelo fabricante da resina. Este pode ser um indício de que a condição ideal de cura não tenha sido atingida.

Nos compósitos houve diminuição gradual na resistência mecânica a tração e flexão com o aumento do teor de resíduo. Os valores de resistência mecânica a tração e flexão do compósito C20 comparativamente aos valores obtidos pela resina pura foram de aproximadamente $47 \%$ e $65 \%$, respectivamente. Esse fato demonstra que os resíduos de MDF no compósito não funcionaram como reforço, e sim como enchimento. Se a adesão das fibras na matriz tivesse sido mais eficiente, e não fosse observada a presença de pull-out, conforme mostrado nas Figura $4 \mathrm{a}$, b, c, as propriedades mecânicas seriam provavelmente superiores.

O alongamento total nas amostras compósitos apresentou comportamento similar ao observado para a resistência mecânica. Como exemplo, os valores de alongamento total para o compósito C20 na tração e na flexão comparativamente aos valores obtidos para resina pura foram de aproximadamente $10 \%$ e $6,7 \%$, respectivamente. Isto mostra que a introdução do enchimento provocou em cada amostra do compósito um menor alongamento e consequentemente um maior módulo de elasticidade quando submetidos aos ensaios de tração e flexão.

\subsection{Absorção de água}

A Tabela 5 apresenta os dados de absorção de água (destilada e do mar) obtidos para a resina pura e para os compósitos.

O índice de absorção de água pelo compósito é considerado baixo mesmo quando comparado a outros compósitos com reforço/enchimento de materiais lignocelulósicos. Compósitos de composição similar aos compósitos $\mathrm{C} 10$ e C20, porém contendo resíduo de madeira em pó, foram estudados por Teixeira et al. ${ }^{[18]}$ e apresentaram os seguintes valores de absorção de água: $1,44 \%$ e 3,77\%, respectivamente para os teores de $10 \%$ e $20 \%$. A diferença entre as absorções deve-se as diferenças entre os resíduos envolvidos na fabricação dos compósitos em questão.

\section{Conclusão}

Conforme resultados obtidos, o compósito proposto apresenta propriedades tecnológicas viáveis para fabricação de peças e estruturas onde as solicitações de esforços mecânicos são menores tais como embalagens, estantes, objetos de decoração, acentos sanitários e prateleiras, além de apresentarem ótimo aspecto visual. Os resultados dos ensaios mecânicos indicam que o resíduo apresentou-se apenas como enchimento ao compósito quando se compara à matriz polimérica de origem, pois não houve melhorias nas propriedades de resistência a tração e flexão provocadas pela baixa adesão entre fibra e matriz observado no ensaio de microscopia eletrônica de varredura assim como pela distribuição aleatória das fibras. Porém, quando se analisa o ganho no módulo de elasticidade, é possível dizer que o resíduo teve a função de reforço na matriz poliéster. Houve influência da concentração de MDF ao compósito reduzindo a resistência mecânica e aumentando o nível de absorção de água pelo compósito fato já esperado devido a ação capilar entre os feixes de fibras e o caráter hidrofílico das fibras. Porém não houve diferença significativa entre os índices de absorção dentre os meios estudados. Este fato sugere que a água do mar não é mais agressiva ao compósito em questão. Vale destacar que não houve degradação ou modificação da fibra de MDF em interação com a matriz poliéster, ou a presença de resíduos de outros gêneros tais como areia, óleo ou outros produtos que comprometessem a cristalização do 
compósito. A formulação mais viável para o compósito foi a C20 por apresentar menores perdas quando comparadas aos demais compósitos com a vantagem de redução da matéria prima utilizada em aplicações industriais.

\section{Referências}

1. Rowell, R. M. (2012). Handbook of wood chemistry and wood composites (2. ed.). Boca Raton: CRC Press.

2. Processo produtivo de chapa de fibra de média densidade (MDF). (2003, Maio). Revista da Madeira, 71. Recuperado em 27 de agosto de 2015, de http://www.remade.com.br/br/ revistadamadeira_materia.php?num $=330 \&$ subject $=$ MDF\&ti tle $=$ Processo

3. Características tecnológicas e aplicações. (2003, Maio). Revista da Madeira, 71. Recuperado em 27 de agosto de 2015, de http:// www.remade.com.br/br/revistadamadeira_materia.php?num $=32$ $8 \&$ subject $=$ Hist $\% \mathrm{C} 3 \% \mathrm{~B} 3$ ria\&title $=$ Caracter $\% \mathrm{C} 3 \% \mathrm{ADsticas} \% 20$ tecnol $\% \mathrm{C} 3 \%$ B3 gicas $\% 20 \mathrm{e} \% 20$ aplica $\% \mathrm{C} 3 \% \mathrm{~A} 7 \% \mathrm{C} 3 \% \mathrm{~B} 5$ es

4. Produção de painéis pode chegar a 12 milhões de $\mathrm{m}^{3} \mathrm{em}$ dez anos. (2010, Julho). Revista da Madeira, 124. Recuperado em 27 de agosto de 2014, de http://www.remade.com.br/br/ revistadamadeira_materia.php?num $=1475 \&$ subject $=$

5. Biazus, A., Hora, A. B., \& Leite, B. G. P. (2010). Panorama de mercado: painéis de madeira. BNDES Setorial, 32, 49-90. Recuperado em 27 de agosto de 2014, de http://www.bndes. gov.br/SiteBNDES/bndes/bndes_pt/Institucional/Publicacoes/ Consulta_Expressa/Tipo/BNDES_Setorial/201009_02.html

6. Moraes, G. S., \& Gordono, F. S. (2015). Análise das vantagens e desvantagens da implantação da ISO 14001 e o Sistema de Gestão Ambiental (SGA). In Anais do $4^{\circ}$ Simpósio de Tecnologia em Meio Ambiente e Recursos Hidricos (pp. 430-441). São Carlos: RiMa. Recuperado em 29 de agosto de 2015, de http:// issuu.com/rimaeditora/docs/anaisjahu

7. Brasil. Ministério do Meio Ambiente - MMA. (2014). Gestão de resíduos. Brasília: Ministério do Meio Ambiente. Recuperado em 20 de agosto de 2014, de http://www.mma. gov.br/responsabilidade-socioambiental/a3p/eixos-tematicos/ gest $\% \mathrm{C} 3 \% \mathrm{~A} 30$-adequada-dos-res $\% \mathrm{C} 3 \% \mathrm{ADduos}$.

8. Calderoni, S. (1999). Os bilhões perdidos no lixo (3. ed.). São Paulo: Humanitas Editora, FFLCH/USP.

9. Abreu, L. B., Mendes, L. M., \& Silva, J. R. M. (2009). Aproveitamento de resíduos de painéis de madeira gerados pela indústria moveleira na produção de pequenos objetos. Revista Árvore, 33(1), 171-177. http://dx.doi.org/10.1590/ S0100-67622009000100018.

10. Casagrande, E. F. J., Maclovia, C. S., Cassilha, A. C., Podlasek, C. L., \& Mengatto, S. N. F. (2004). Indústria moveleira e resíduos sólidos: considerações para o equilíbrio ambiental. Revista Educação \& Tecnologia, 8, 209-228. Recuperado em 27 de agosto de 2014, de http://revistas.utfpr.edu.br/pb/index. $\mathrm{php} /$ revedutec-ct/article/view/1142/739

11. Ferreira, S. D., Altafini, C. R., Perondi, D., \& Godinho, M. (2015). Pyrolysis of Medium Density Fiberboard (MDF) wastes in a screw reactor. Energy Conversion and Management, 92, 223-233. http://dx.doi.org/10.1016/j.enconman.2014.12.032.

12. Farage, R. M. O., Rezende, A. A. P., Silva, C. M., Nunes, W. G., Carneiro, A. C. O., Vieira, D. B., \& Rodrigues, C. L. S. (2012). Avaliação do potencial de aproveitamento energético dos resíduos de madeira e derivados gerados em fábricas do polo moveleiro de Ubá - MG. Revista Ciência Florestal, 23(1), 203-212. http://dx.doi.org/10.5902/198050988454.

13. Hedlund, T. A. (2013). A redução dos impactos ambientais como proposta para o desenvolvimento de luminária a partir de resíduos de MDF (Monografia). Universidade Regional do
Noroeste do Estado do Rio Grande do Sul, Ijuí. Recuperado em 27 de agosto de 2014, de http://bibliodigital.unijui.edu. br:8080/xmlui/handle/123456789/1772

14. Hillig, E., Iwakiri, S., Andrade, M. Z., \& Zattera, J. (2008). Characterization of composites made from high density polythylene (HDPE) and furniture industry sawdust. Revista Árvore, 32(2), 299-310. Recuperado em 27 de agosto de 2014, de http://www. scielo.br/scielo.php?pid=S0100-67622008000200013\&amp; script $=$ sci_arttext

15. Jung, C. F., Rosa, F. P., \& Sporket, F. (2014). Desenvolvimento de absorvedores de radiações eletromagnéticas a partir de resíduos de indústrias alimentícias e moveleiras: uma alternativa sustentável. In Anais do XII Simpósio de Excelência em Gestão e Tecnologia (pp. 1-16). Resende: AEDB. Recuperado em 27 de agosto, de http://www.aedb.br/seget/arquivos/artigos14/16120137. pdf

16. Piekarski, C. M., Francisco, A. C., Luz, L. M., Alvarenga, T. H. D. P., \& Bittencourt, J. V. M. (2014). Environmental profile analysis of MDF panels production: study in a brazilian technological condition. Cerne, 20(3), 409-418. Recuperado em 27 de agosto de 2014, de http://www.scielo.br/pdf/cerne/ v20n3/a10v20n3.pdf

17. Silva, A. F., \& Figueiredo, C. F. (2010). Reaproveitamento de resíduos de MDF da indústria moveleira. Design \& Tecnologia, 2, 77-87. Recuperado em 27 de agosto de 2014, de http:// www.pgdesign.ufrgs.br/designetecnologia/index.php/det/ article/view/49

18. Teixeira, M. G., \& César, S. F. (2006). Produção de compósito com resíduo de madeira no contexto da ecologia industrial. Revista Madeira Arquitetura \& Engenharia, 7(19), 1-15. Recuperado em 27 de agosto de 2014, de http://madeira.set. eesc.usp.br/article/view/219

19. Teixeira, A. O. (2011). Avaliação de oportunidades de implementação de $P+L$ em uma marcenaria de pequeno porte e proposta de ecoproduto (Dissertação de mestrado). Universidade de Santa Cruz do Sul, Capão da Canoa. Recuperado em 27 de agosto de 2014, de http://www.unisc.br/portal/upload/ com_arquivo/dini_final.pdf

20. Weber, C., \& Iwakiri, S. (2015). Utilização de resíduos de compensados, MDF e MDP para produção de painéis aglomerados. Ciência Florestal, 25(2), 405-413. Recuperado em 27 de agosto de 2014, de http://www.scielo.br/pdf/cflo/ v25n2/0103-9954-cflo-25-02-00405.pdf

21. World Health Organization. International Agency for Research on Cancer. (2006). IARC Monographs on the Evaluation of Carcinogenic Risks to Humans (Vol. 88). Lyon: IARC Working Group. Recuperado em 27 de agosto de 2014, de http:// monographs.iarc.fr/ENG/Monographs/vol88/mono88.pdf

22. Dutkiewicz, J. (1983). Hydrolytic degradation of cured ureaformaldehyde resin. Journal of Applied Polymer Science, 28, 3313-3320. http://dx.doi.org/10.1002/app.1983.070281101.

23. Rio Grande do Sul. Governo do Estado. Fundação Estadual de Proteção Ambiental Henrique Luiz Roessler - FEPAM. (2012, 15 de fevereiro). Portaria $n^{\circ}$ 009/2012, de 08 de fevereiro de 2012. Dispõe sobre o regramento para o uso de derivados de madeira, em especial MDP e MDF (Medium Density Fiberboard e Medium Density Particleboard), não contaminados, como combustivel alternativo/principal. Diário Oficial do Estado, Porto Alegre. Recuperado em 27 de agosto de 2014, de http:// www.proamb.com.br/downloads/d5mhjg.pdf

24. Klyosov, A. A. (2007). Wood-plastic composites. New Jersey: John Wiley \& Sons.

25. Rodolfo, A., Jr., \& John, V. M. (2006). Desenvolvimento de PVC reforçado com resíduos de Pinus para substituir madeira convencional em diversas aplicações. Polímeros: Ciência e 
Tecnologia, 16(1), 1-11. http://dx.doi.org/10.1590/S010414282006000100005 .

26. Wakelyn, N. T., \& Young, P. R. (2003). Crystallinity index of poly(ethylene terephthalate) by x-ray diffractometry and differential scanning calorimetry. Journal of Applied Polymer Science, 10(10), 1421-1428. http://dx.doi.org/10.1002/ app.1966.070101004.

27. American Society for Testing and Materials-ASTM. (2003) ASTM D638-03 - Standard test method for tensile properties of plastic. West Conshohocken: ASTM.

28. American Society for Testing and Materials - ASTM. (2001). ASTM D790 Standard Test Methods: Flexural properties of unreinforced and reinforced plastics and electrical insulating materials. West Conshohocken: ASTM.

29. American Society for Testing and Materials-ASTM. (2010). ASTM D570-98 - Standard Test Method for Water Absorption of Plastics. West Conshohocken: ASTM.

30. Rabiej, S. (1991). A comparison of two X-ray diffraction procedures for crystallinity determination. European Polymer Journal, 27(9), 947-954. http://dx.doi.org/10.1016/00143057(91)90038-P.

31. Xing, C., Zhang, S. Y., \& Deng, J. (2004). Effect of wood acidity and catalyst on UF resin gel time. Holzforschung, 58(4), 408-412. http://dx.doi.org/10.1515/HF.2004.061.

32. Belini, U. L., Tomazello, M., Fo., Chagas, M. P., \& Oliveira, J. T. D. S. (2008). Alterações na estrutura anatômica da madeira de cavacos de Eucalyptus grandis em três condições de desfibramento para a confecção de painéis MDF. Revista Árvore, 32(3), 523-532. http://dx.doi.org/10.1590/S010067622008000300013 .

33. Associação Brasileira de Celulose e Papel - BRACELPA (2014). Dados do setor. São Paulo: BRACELPA. Recuperado em 27 de agosto de 2014, de http://bracelpa.org.br/bra2/sites/ default/files/estatisticas/booklet.pdf
34. Vital, M. H. F. (2007). Impacto ambiental de florestas de eucalipto. Revista do BNDES, 14(28), 235-276. Recuperado em 27 de agosto de 2014, de http://www.bndes.gov.br/SiteBNDES/ export/sites/default/bndes_pt/Galerias/Arquivos/conhecimento/ revista/rev2808.pdf

35. Silva, J. C. (2014). Eucalipto. Desfazendo mitos e preconceitos. Presidente Prudente: Fazendas Floresta. Recuperado em 27 de agosto de 2014, de http://fazendasfloresta.com.br/materia3. asp

36. Brasil. Congresso. (2014). Seguridade discute problemas causados pela monocultura do eucalipto na Bahia. Brasília: Câmara dos Deputados. Recuperado em 9 de setembro de 2015, de http://www2.camara.leg.br/camaranoticias/noticias/471567. html

37. Benjamin, C. A. (2006). Estudo da estrutura anatômica e das propriedades fisicas e mecânicas da madeira Corymbia (Eucalyptus) citriodora e Eucalyptus grandis. Brasília. Recuperado em 27 de agosto de 2014, de http://bdtd.ibict. $\mathrm{br} /$ vufind/Record/UNSP_46d0c9c76e7c9d73e29d7220993b $\mathrm{d} 8 \mathrm{e} 2$

38. Zegarra, B. E. V. (2011). Caracterização da estrutura anatômica e da densidade do lenho de árvores de Pinus taeda e efeito nas propriedades tecnológicas dos painéis OSB (Dissertação de mestrado). Universidade de São Paulo, Piracicaba. http:// dx.doi.org/10.11606/D.11.2011.tde-22122011-084728.

39. Hu, X.-P., \& Hsieh, Y.-L. (1996). Crystalline structure of developing cotton fibers. Journal of Polymer Science. Part B, Polymer Physics, 34(8), 1451-1459. http://dx.doi.org/10.1002/ (SICI)1099-0488(199606)34:8<1451::AID-POLB8>3.0.CO;2-V.

Enviado: Set. 13, 2014

Revisado: Set. 23, 2015

Aceito: Fev. 15, 2016 